\title{
Prevalence and Trends of Vitamin D Deficiency among Iranian Adults: A Longitudinal Study from 2001-2013
}

\author{
Hossein Khosravi-Boroujeni ${ }^{1,2}$, Nizal SARrafZAdEGAN ${ }^{3, *}$, Masoumeh SAdEGHI ${ }^{4}$, \\ Hamidreza RoOHAFZA ${ }^{4}$, Shu-Kay NG ${ }^{1,2}$, Ali PourMOGADDAS ${ }^{3}$ and Faruk AHMED ${ }^{1,2}$ \\ ${ }^{1}$ School of Medicine, Griffith University, QLD, Australia \\ ${ }^{2}$ Menzies Health Institute Queensland, QLD, Australia \\ ${ }^{3}$ Isfahan Cardiovascular Research Center, Cardiovascular Research Institute, \\ Isfahan University of Medical Sciences, Isfahan, Iran \\ ${ }^{4}$ Cardiac Rehabilitation Research Center, Cardiovascular Research, Iran
}

(Received March 22, 2017)

\begin{abstract}
Summary Vitamin D deficiency/insufficiency is currently considered to be a re-emerging public health problem globally. This study was designed to determine the prevalence of vitamin D deficiency and insufficiency and to investigate its trend from 2001 to 2013 in a longitudinal study of Iranian adults. This study was part of a population-based, longitudinal ongoing study of Iranian healthy adults aged $35 \mathrm{y}$ and older at baseline. Serum vitamin D level was assessed in a sub-sample of 370 subjects, who were apparently healthy at the time of recruitment in 2001 and were free from MetS, in three phases (2001, 2007 and 2013) during the 12-y study period. Adjusted prevalence and trend of vitamin D deficiency were calculated. Mean serum vitamin D levels increased over the time of the study $(52.12,54.27$ and $62.28 \mathrm{nmol} / \mathrm{L}$, respectively) and the prevalence of vitamin D deficiency decreased (30.5, 27.0 and 24.4, respectively). However, the prevalence of vitamin D insufficiency did not change over this time period. The risk of vitamin D deficiency decreased significantly in 2007 [OR: 0.73 (95\% CI: 0.53, 0.99)] and 2013 [OR: 0.50 (95\% CI: 0.36, 0.70)] compared to the baseline. The present study demonstrated some improvement in serum vitamin D levels, while the prevalence of vitamin D inadequacy was still high. Considering the possible health consequences of vitamin D deficiency, there is an urgent need for developing population-wide strategies, such as supplementation and fortification, to prevent or control vitamin D deficiency.
\end{abstract}

Key Words vitamin D deficiency, vitamin D insufficiency, prevalence, longitudinal study

Vitamin D deficiency was identified as a public health problem more than two hundred years ago, during the Industrial Revolution (1). The problem declined as a result of the discovery of vitamin $\mathrm{D}$ and increased intake of vitamin D. However, as a consequence of modern lifestyles, more indoor activities and the inadequate amount of vitamin D in most people's diets (2), the number of individuals with low serum 25-hydroxyvitamin D (25(OH)D) levels has been increasing (3).

Vitamin D has long been recognized for its role in the regulation of calcium and phosphorus homeostasis and bone health (4). Moreover, there is also an increasing body of evidence that has found an association between vitamin D deficiency and other health problems (5). For example, the risk of all-cause and cardiovascular mortality was reported higher in people with a lower level of serum 25(OH)D (6-8). Although the optimal serum level of $25(\mathrm{OH}) \mathrm{D}$ in humans is not fully recognised, several expert groups have considered levels of less than $25 \mathrm{nmol} / \mathrm{L}$ to be a 'deficiency' and 25-50 nmol/L to be an 'insufficiency' $(9,10)$.

\footnotetext{
*To whom correspondence should be addressed.

E-mail: nsarrafzadegan@gmail.com
}

Due to the potential health problems related to vitamin D deficiency and its increasing prevalence, vitamin $\mathrm{D}$ deficiency/insufficiency is currently recognized as a reemerging public health problem at the global level (11). It is estimated that more than one billion people are vitamin D deficient/insufficient (12). For example, the prevalence of vitamin D deficiency was $36 \%$ in young adults in the United States (13), while studies in Australia have also reported a high prevalence of vitamin D deficiency (33\% and $40 \%$ in men and women respectively), despite the high levels of sunshine (14). However, the situation seems to be worse in Middle-Eastern countries, despite the sunny climate throughout the year (15). The prevalence of vitamin $\mathrm{D}$ deficiency/insufficiency in adults was reported as $61.4 \%$ and $87.8 \%$ in Kuwait and Saudi Arabia respectively $(16,17)$. Although there is no national level data on vitamin D deficiency in Iran, several small scale studies reported a prevalence of $44.2 \%$ to $72.3 \%$ in adults $(18,19)$. Further, there are few longitudinal studies which have investigated the trends in the prevalence of vitamin D deficiency (20). Given the importance of adequate 25(OH)D levels and chronic disease prevention, this study aimed to determine the current prevalence of vitamin D deficiency and insufficiency, 
Table 1. Serum 25(OH)D levels, based on individual characteristics, from 2001 to 2013.

\begin{tabular}{|c|c|c|c|c|c|c|c|c|}
\hline & \multirow{2}{*}{$n(2001)$} & \multicolumn{2}{|c|}{2001} & \multicolumn{2}{|c|}{2007} & \multicolumn{2}{|c|}{2013} & \multirow{2}{*}{$p$ value } \\
\hline & & Mean & (SD) & Mean & (SD) & Mean & (SD) & \\
\hline Total & 370 & 52.12 & $(44.81)$ & 54.27 & $(42.93)$ & 62.28 & $(46.26)$ & $<0.05$ \\
\hline \multicolumn{9}{|l|}{ Sex } \\
\hline Male & 216 & 50.66 & $(40.12)$ & 51.38 & $(40.71)$ & 57.99 & $(41.00)$ & \multirow[t]{2}{*}{$<0.05$} \\
\hline Female & 154 & $\begin{array}{l}54.19 \\
\text { NS }\end{array}$ & $(51.12)$ & $\begin{array}{l}56.34 \\
\text { NS }\end{array}$ & $(45.89)$ & $\begin{array}{l}68.28 \\
\text { NS }\end{array}$ & (51.99) & \\
\hline \multicolumn{9}{|l|}{ Age categories } \\
\hline $35-45$ & 198 & 48.88 & $(39.96)$ & 50.48 & (39.21) & 56.26 & (39.78) & \multirow[t]{4}{*}{$<0.05$} \\
\hline $45-55$ & 109 & 51.85 & $(42.70)$ & 55.14 & $(29.02)$ & 56.26 & \multirow{3}{*}{$\begin{array}{l}(29.44) \\
(78.10)\end{array}$} & \\
\hline \multirow[t]{2}{*}{$>65$} & 63 & 62.63 & $(82.94)$ & 64.58 & $(80.26)$ & 77.80 & & \\
\hline & & NS & & NS & & NS & & \\
\hline \multicolumn{9}{|l|}{ BMI } \\
\hline$<25$ & 141 & 48.91 & $(34.10)$ & 52.30 & $(45.95)$ & 62.35 & $(58.16)$ & \multirow[t]{4}{*}{$<0.05$} \\
\hline $25-30$ & 152 & 53.81 & $(53.01)$ & 56.79 & $(43.02)$ & 59.75 & \multirow{3}{*}{$\begin{array}{l}(42.38) \\
(48.27)\end{array}$} & \\
\hline \multirow[t]{2}{*}{$>30$} & 77 & 50.57 & $(45.27)$ & 52.27 & $(38.91)$ & 62.65 & & \\
\hline & & NS & & NS & & NS & & \\
\hline \multicolumn{9}{|l|}{ Region } \\
\hline Rural & 79 & 45.95 & (39.73) & 54.56 & $(36.44)$ & 64.92 & $(46.75)$ & \multirow[t]{3}{*}{$<0.05$} \\
\hline \multirow[t]{2}{*}{ Urban } & 291 & 53.78 & $(46.05)$ & 54.19 & $(44.69)$ & 60.55 & \multirow[t]{2}{*}{ (46.05) } & \\
\hline \multirow{2}{*}{\multicolumn{9}{|c|}{ Education level }} \\
\hline & & & & & & & & \\
\hline Illiterate & 67 & 60.53 & $(56.15)$ & 65.48 & $(51.98)$ & 73.13 & $(54.35)$ & \multirow[t]{4}{*}{$<0.05$} \\
\hline Primary school & 135 & 49.61 & $(44.50)$ & 52.61 & $(39.15)$ & 65.64 & & \\
\hline \multirow[t]{2}{*}{$>$ primary school } & 168 & 50.83 & $(39.27)$ & 51.29 & $(41.62)$ & 55.06 & $(41.92)$ & \\
\hline & & NS & & NS & & NS & & \\
\hline Marriage & & & & & & & & \\
\hline Single & 16 & 58.92 & $(48.82)$ & 72.13 & $(52.21)$ & 79.38 & $(42.76)$ & $<0.05$ \\
\hline Married & 354 & 51.84 & $(44.77)$ & 53.50 & $(42.52)$ & 61.53 & $(46.09)$ & \\
\hline & & NS & & NS & & NS & & \\
\hline Smoking & & & & & & & & \\
\hline Smoker & 93 & 47.17 & $(36.54)$ & 51.48 & $(42.23)$ & 59.13 & $(46.48)$ & $<0.05$ \\
\hline Non smoker & 277 & 53.75 & $(47.43)$ & 55.32 & $(43.02)$ & 63.47 & $(46.12)$ & \\
\hline & & NS & & NS & & NS & & \\
\hline Occupation & & & & & & & & \\
\hline Outdoor & 96 & 58.19 & $(42.13)$ & 62.39 & $(52.51)$ & 67.76 & (33.97) & $<0.05$ \\
\hline Indoor & 274 & 56.00 & $(98.65)$ & 56.95 & $(90.37)$ & 66.01 & $(90.93)$ & \\
\hline & & NS & & NS & & NS & & \\
\hline Dietary sources of vi & & & & & & & & \\
\hline Low & 223 & 57.99 & (68.39) & 59.60 & $(84.82)$ & 61.11 & $(54.80)$ & $<0.05$ \\
\hline High & 147 & 54.49 & $(64.62)$ & 58.23 & $(60.98)$ & 72.91 & (69.23) & \\
\hline & & NS & & NS & & NS & & \\
\hline
\end{tabular}

Data are means (standard deviation) of serum 25(OH)vitamin D.

${ }^{1}$ butter, cream, high fat dairy products, fish, liver and whole eggs.

and to examine its trend in a longitudinal study of Iranian adults with three times assessments from 2001 to 2013.

\section{METHODS AND MATERIALS}

Study design. This study was completed as part of a population-based, longitudinal, ongoing study of healthy Iranian adults aged $35 \mathrm{y}$ and older at the time of recruitment in 2001, known as the Isfahan Cohort Study (ICS) (21). Individuals were selected from three provinces in central Iran, using the multistage random sampling method. ICS aimed to determine the impact of various risk factors, including individual and environmental risk factors, on the incidence of cardiovascular diseases. Detailed information regarding ICS, including sampling, rationale and results, was presented else- where $(21,22)$. In the current study, vitamin D levels were assessed in subjects who were free of MetS in 2001 and if their frozen serum samples were available in 2001, 2007 and 2013. Thus, using the above criteria, 370 subjects met the selection criteria for the current study. The ethics committees of the Isfahan Cardiovascular Research Institute (ICRI) and Griffith University approved the current study.

Measurements. After obtaining informed written consent, an interview was conducted by trained interviewers. A validated questionnaire, comprising questions on socio-demographic characteristics and related lifestyle behaviours, including smoking, physical activity, and nutritional habits, was applied. Anthropometric parameters were measured using standard protocols. Body mass index (BMI) was calculated by dividing the 
Table 2. Baseline prevalence of vitamin D deficiency and insufficiency among Iranian adults by characteristics of study population.

\begin{tabular}{|c|c|c|c|c|c|c|c|}
\hline & \multicolumn{6}{|c|}{ Serum $25(\mathrm{OH})$ vitamin D categories } & \multirow{3}{*}{$p$ value } \\
\hline & \multicolumn{2}{|c|}{$\begin{array}{c}\text { Deficient } \\
<25 \mathrm{nmol} / \mathrm{L}\end{array}$} & \multicolumn{2}{|c|}{$\begin{array}{c}\text { Insufficient } \\
25-50 \mathrm{nmol} / \mathrm{L}\end{array}$} & \multicolumn{2}{|c|}{$\begin{array}{c}\text { Sufficient } \\
>50 \mathrm{nmol} / \mathrm{L}\end{array}$} & \\
\hline & $n$ & $(\%)$ & $n$ & $(\%)$ & $n$ & $(\%)$ & \\
\hline Total & 123 & $(33.4 \%)$ & 106 & $(28.8 \%)$ & 141 & $(37.8 \%)$ & \\
\hline \multicolumn{8}{|l|}{ Sex } \\
\hline Male & 69 & $(31.9 \%)$ & 62 & $(28.7 \%)$ & 85 & $(39.4 \%)$ & 0.50 \\
\hline Female & 54 & $(35.1 \%)$ & 44 & $(28.6 \%)$ & 56 & $(36.4 \%)$ & \\
\hline \multicolumn{8}{|l|}{ Age categories } \\
\hline $35-45$ & 74 & $(36.4 \%)$ & 54 & $(26.8 \%)$ & 73 & $(36.9 \%)$ & 0.99 \\
\hline $45-55$ & 32 & $(28.4 \%)$ & 42 & $(37.6 \%)$ & 39 & $(33.9 \%)$ & \\
\hline$>55$ & 21 & $(38.5 \%)$ & 13 & $(23.0 \%)$ & 22 & $(38.5 \%)$ & \\
\hline \multicolumn{8}{|l|}{ BMI } \\
\hline$<25$ & 40 & $(28.4 \%)$ & 48 & $(34.0 \%)$ & 53 & $(37.6 \%)$ & 0.26 \\
\hline $25-30$ & 59 & $(38.8 \%)$ & 37 & $(24.3 \%)$ & 56 & $(36.8 \%)$ & \\
\hline$>30$ & 24 & $(31.2 \%)$ & 21 & $(27.3 \%)$ & 32 & $(41.6 \%)$ & \\
\hline \multicolumn{8}{|l|}{ Region } \\
\hline Rural & 89 & $(30.6 \%)$ & 86 & $(29.6 \%)$ & 116 & $(39.9 \%)$ & 0.11 \\
\hline Urban & 34 & $(43.0 \%)$ & 20 & $(25.3 \%)$ & 25 & $(31.6 \%)$ & \\
\hline \multicolumn{8}{|l|}{ Education level } \\
\hline Illiterate & 22 & $(32.8 \%)$ & 16 & $(23.9 \%)$ & 29 & $(43.3 \%)$ & 0.48 \\
\hline Primary school & 52 & $(38.5 \%)$ & 39 & $(28.9 \%)$ & 44 & $(32.6 \%)$ & \\
\hline$>$ primary school & 49 & $(29.2 \%)$ & 51 & $(30.4 \%)$ & 68 & $(40.5 \%)$ & \\
\hline \multicolumn{8}{|l|}{ Marriage } \\
\hline Single & 4 & $(28.6 \%)$ & 3 & $(21.4 \%)$ & 7 & $(50.0 \%)$ & 0.11 \\
\hline Married & 119 & $(33.4 \%)$ & 103 & $(28.9 \%)$ & 134 & $(37.6 \%)$ & \\
\hline \multicolumn{8}{|l|}{ Smoking } \\
\hline Smoker & 32 & $(34.8 \%)$ & 23 & $(25.0 \%)$ & 37 & $(40.2 \%)$ & 0.29 \\
\hline Non smoker & 92 & $(32.9 \%)$ & 82 & $(29.6 \%)$ & 104 & $(37.5 \%)$ & \\
\hline \multicolumn{8}{|l|}{ Occupation $^{1}$} \\
\hline Outdoor & 44 & $(33.6 \%)$ & 34 & $(26.0 \%)$ & 53 & $(40.5 \%)$ & 0.34 \\
\hline Indoor & 79 & $(33.1 \%)$ & 72 & $(30.1 \%)$ & 88 & $(36.8 \%)$ & \\
\hline \multicolumn{8}{|c|}{ Dietary sources of vitamin D } \\
\hline Low & 77 & $(35.6 \%)$ & 53 & $(24.5 \%)$ & 86 & $(39.8 \%)$ & 0.11 \\
\hline High & 46 & $(29.9 \%)$ & 53 & $(34.4 \%)$ & 55 & $(35.7 \%)$ & \\
\hline
\end{tabular}

${ }^{1}$ Governmental jobs and housework were considered indoor and self-employed, and not working and retired were considered outdoor occupations.

weight in kilograms by height in meters squared. Blood samples were collected after $12 \mathrm{~h}$ of fasting and were immediately frozen at $-80^{\circ} \mathrm{C}$ for future studies. The serum 25(OH)D levels, as the best measure of body vitamin D status (23), were measured using the Enzyme Linked Immune Sorbent Assay (ELISA; Euroimmun AG, Luebeck, Germany). For the current study, the frozen serum samples were used to measure $25(\mathrm{OH}) \mathrm{D}$, as it is very stable in serum when kept frozen (24).

The dietary intake of participants was assessed using a validated 49-item food frequency questionnaire (FFQ) $(25,26)$ that determined the consumption of each food item during the previous year. The main dietary sources of vitamin D, including oily fish, butter, margarine, eggs, beef and liver were considered as dietary intake of vitamin $\mathrm{D}$, which was assessed by summing up the frequency of consumption of those foods. Based on the median intake, individuals with a three or more times per week consumption of vitamin D sources were defined as high consumers. Serum vitamin D levels were categorized based on the most accepted cut-off points for deficiency, insufficiency and sufficiency $(<25 \mathrm{nmol} / \mathrm{L}$,
25-50 nmol/L and $>50 \mathrm{nmol} / \mathrm{L}$ respectively) (9).

Statistical analysis. Variables were presented as the mean (standard deviation) or percentage. The frequency of distribution of participants among these three groups was examined using Chi-square or the Fisher Exact test, where appropriate. Changes in serum vitamin D levels from 2001 to 2013 were tested using repeated measures ANOVA. Analyses were stratified by gender, age, BMI, region, education levels, occupation and dietary intake of vitamin D. The Generalized Estimating Equation (GEE) (27) was used to investigate the age-adjusted odds ratios for the prevalence and trend of vitamin $\mathrm{D}$ deficiency and insufficiency from 2001 to 2013. In this model, the logistic regression and the $\operatorname{AR}(1)$ working correlation matrix have been used. To control the effect of potential confounders on the trends of vitamin D deficiency and insufficiency, age, BMI, region, season of blood collection and dietary intake of vitamin $\mathrm{D}$ were adjusted in the model. The predicted value was determined in GEE and the prevalence for each period was calculated. The SPSS statistical software package version 22 (SPSS Inc., Chicago, IL) was used for statistical analysis. 
Table 3. Prevalence of vitamin D deficiency and insufficiency among Iranian adults during 12-y study period from 2001 to 2013 , separated by sex.

\begin{tabular}{|c|c|c|c|c|}
\hline & Total & Male & Female & $p$ value $^{1}$ \\
\hline \multicolumn{5}{|c|}{ Vitamin D deficiency } \\
\hline 2001 & $30.5 \%$ & $29.9 \%$ & $31.2 \%$ & \multirow[t]{3}{*}{$<0.001$} \\
\hline 2007 & $27.0 \%$ & $26.7 \%$ & $27.5 \%$ & \\
\hline 2013 & $24.4 \%$ & $24.5 \%$ & $24.2 \%$ & \\
\hline \multicolumn{5}{|c|}{ Vitamin D insufficiency } \\
\hline 2001 & $31.7 \%$ & $31.7 \%$ & $31.7 \%$ & \multirow[t]{3}{*}{0.05} \\
\hline 2007 & $30.7 \%$ & $30.9 \%$ & $30.5 \%$ & \\
\hline 2013 & $30.2 \%$ & $30.8 \%$ & $29.5 \%$ & \\
\hline \multicolumn{5}{|c|}{ Vitamin D deficiency/insufficiency } \\
\hline 2001 & $62.0 \%$ & $61.6 \%$ & $62.6 \%$ & \multirow[t]{3}{*}{$<0.001$} \\
\hline 2007 & $57.9 \%$ & $57.7 \%$ & $58.2 \%$ & \\
\hline 2013 & $53.9 \%$ & $53.9 \%$ & $53.9 \%$ & \\
\hline \multicolumn{5}{|c|}{ Vitamin D sufficiency } \\
\hline 2001 & $37.9 \%$ & $38.4 \%$ & $37.3 \%$ & \multirow[t]{3}{*}{$<0.001$} \\
\hline 2007 & $42.1 \%$ & $42.3 \%$ & $41.8 \%$ & \\
\hline 2013 & $46.1 \%$ & $46.0 \%$ & $46.1 \%$ & \\
\hline
\end{tabular}

${ }^{1}$ Adjusted for age, BMI, region, education levels, occupation, season of blood collection and dietary intake of vitamin D.

Table 4. Odds ratios of for prevalence of vitamin D deficiency and insufficiency according to study period in Iranian adults, using longitudinal data from 2001 to $2013 .{ }^{1}$

\begin{tabular}{|c|c|c|c|c|c|c|}
\hline & \multicolumn{2}{|c|}{ Total $^{2}$} & \multicolumn{2}{|c|}{ Male } & \multicolumn{2}{|c|}{ Female } \\
\hline & Odds ratio & $95 \% \mathrm{CI}$ & Odds ratio & $95 \% \mathrm{CI}$ & Odds ratio & $95 \% \mathrm{CI}$ \\
\hline \multicolumn{7}{|c|}{ Vitamin D deficiency } \\
\hline 2001 & 1.00 & - & 1.00 & - & 1.00 & - \\
\hline 2007 & 0.73 & $0.53,0.99$ & 0.76 & $0.50,1.16$ & 0.68 & $0.42,0.98$ \\
\hline 2013 & 0.50 & $0.36,0.70$ & 0.45 & $0.28,0.71$ & 0.56 & $0.34,0.93$ \\
\hline \multicolumn{7}{|c|}{ Vitamin D insufficiency } \\
\hline 2001 & 1.00 & - & 1.00 & - & 1.00 & - \\
\hline 2007 & 0.89 & $0.66,1.19$ & 1.17 & $0.79,1.74$ & 0.61 & $0.38,0.96$ \\
\hline 2013 & 0.58 & $0.44,0.78$ & 0.72 & $0.48,1.05$ & 0.44 & $0.28,0.69$ \\
\hline
\end{tabular}

${ }^{1}$ Adjusted for age, BMI, region and vitamin D intake.

${ }^{2}$ Adjusted for age, gender, BMI, region and vitamin D intake in total.

\section{RESULTS}

Examining the serum vitamin D levels from 2001 to 2013 revealed that mean serum $25(\mathrm{OH}) \mathrm{D}$ levels were $52.1 \mathrm{nmol} / \mathrm{L}, 54.3 \mathrm{nmol} / \mathrm{L}$ and $62.3 \mathrm{nmol} / \mathrm{L}$ in 2001 , 2007 and 2013, respectively (Table 1). The results identified an increasing trend in serum vitamin D levels over the 12-y period, even when classified according to gender, age, BMI, region, education levels, marital and smoking status, occupation and dietary intake of vitamin D. When comparing serum vitamin D levels separately at different points in time of data collection, there were no statistically significant differences in serum vitamin D levels by various socio-demographic groups, such age, BMI and gender. The prevalence of vitamin D insufficiency or deficiency at baseline did not differ significantly between different categories of various sociodemographic factors. Further, it was not significantly different between groups with low and high vitamin D intake (Table 2).

The prevalence of vitamin D deficiency, insufficiency and adequacy were adjusted for age, BMI, region, season of blood collection and dietary intake of vitamin
D (Table 3). The results identified a significant decreasing trend in the prevalence of vitamin D deficiency in both males and females from 2001 to 2013 (6.1\%, p value $<0.001)$, while vitamin $\mathrm{D}$ sufficiency increased significantly during this period $(8.2 \%, p$ value $<0.001)$ from 2001 to 2013. However, the decreasing trend in the prevalence of vitamin D insufficiency approached a significant level. The overall prevalence of vitamin D deficiency/insufficiency also decreased by $8.1 \%$ (7.6\% in males and $8.8 \%$ in females). Although the prevalence of vitamin D inadequacy (combined insufficiency and deficiency) decreased over the 12-y period, the prevalence of vitamin D deficiency and insufficiency were still high in 2001, 2007 and 2013 (62.0\%, 57.9\% and 53.9\%, respectively).

Table 4 compares the risk of being vitamin $\mathrm{D}$ deficient and insufficient in the years 2007 and 2013 with 2001. For the studied population, the risk of vitamin D deficiency decreased significantly in 2007 [OR: 0.73 (95\% CI: 0.53, 0.99)] and 2013 [OR: 0.50 (95\% CI: $0.36,0.70)]$, while the risk of vitamin D insufficiency decreased significantly only in 2013 [OR: 0.58 (95\% CI: $0.44,0.78)]$. In females, the risk of vitamin D deficiency 
also decreased in 2007 and 2013, while in males, the reduced risk was significant only in 2013. Although the risk of vitamin D insufficiency decreased significantly in females in 2007 [OR: 0.61 (95\% CI: 0.38, 0.96)] and 2013 [OR: 0.44 (95\% CI: 0.28, 0.69)], no significant risk was found in males. Analysing the association between dietary intake of vitamin $\mathrm{D}$ and serum vitamin D levels failed to uncover any significant relationship (data not shown).

\section{DISCUSSION}

This study provided an insight into the prevalence and trends of vitamin D deficiency and insufficiency among Iranian adults from 2001 to 2013. The present longitudinal study revealed some important findings. First, this study identified a decreasing trend in vitamin $\mathrm{D}$ deficiency and an increasing trend in vitamin $\mathrm{D}$ adequacy over the 12-y period since 2001. Moreover, when the distribution of serum vitamin D levels was examined at different points in time, there was a positive shift from 2001 to 2013. Furthermore, the results also indicated that vitamin D deficiency and insufficiency are still highly prevalent among the studied population. These results add to the expanding data suggesting that vitamin D inadequacy is a highly prevalent situation globally, as well as in the Middle Eastern countries (16-19, 28 ). Previous studies in Iran also reported a high prevalence of vitamin D deficiency and insufficiency (20, 29, 30); however, to the best of our knowledge, no cohort study has reported changes in vitamin D levels over time in Iran or in any of the Middle East countries.

The present study also reported a decreased risk of vitamin D deficiency over the last $12 \mathrm{y}$. This apparent improvement in vitamin D status could be the result of several possible factors. First, in recent years, the general population and medical professionals have become more aware of the problem of vitamin D deficiency and its health consequences, including skeletal disorders and chronic diseases. Moreover, medical doctors are more likely to screen for vitamin D status, and there is a higher possibility for diagnosis of vitamin D deficiency and a greater likelihood to recommend the use of vitamin D supplementation. A study in the same district of Iran compared results on vitamin D deficiency with previous studies in Iran and claimed that the prevalence of vitamin D deficiency is an increasing trend (20). However, because of the cross-sectional design of the compared studies, different methods of serum 25(OH)D assessment, different population and different definitions for vitamin $\mathrm{D}$ deficiency among studies, this assumption may not be accurate.

Vitamin D deficiency could be attributable to low dietary intake of vitamin D and low sun exposure. However, the present study showed no association between serum vitamin D levels and dietary intake of vitamin D. This result is expected because there are limited foods that naturally contain vitamin $\mathrm{D}$, and almost all foods have less than $400 \mathrm{IU}$ of vitamin D per $100 \mathrm{~g}$ edible portion. The best sources of vitamin D are oily fish, cod liver oil, beef liver and egg yolk (31). Alternatively, during sunlight exposure, ultraviolet (UV) photons are absorbed by the epidermal and dermal cells and produce vitamin $\mathrm{D}_{3}$ from its pre-vitamin (32).

Despite ample sunshine, Iran is among the countries with the highest prevalence of vitamin D deficiency and insufficiency (11). The present study showed that over half of the studied population were vitamin D deficient or insufficient. This is, in large part, elucidated by restricted sun exposure, skin pigmentation and social factors (28). Skin pigmentation prevents UV absorption and thus the skin produces less vitamin D in the body (33). Likewise, more popular use of sunscreen may also reduce the cutaneous synthesis of vitamin D (34). On the other hand, sun-seeking behaviour is not popular among the Iranian population because of the hot and dry climate and to prevent pigmentation, as fair skin is considered an aspect of beauty in this society (11). Moreover, many people avoid UV irradiation because of the growing awareness that sunshine may lead to premature aging and cancers (35). Skin coverage is another factor affecting vitamin D synthesis and leading to vitamin D deficiency (36), and is common in Iran because of cultural and religious considerations. However, in this study there are no data to support this statement.

Generally, urbanization is another reason for low serum vitamin D levels, as people spend most of their time indoors, compared to rural citizens. As a result of increased population, a higher percentage of the population live in apartments, also diminishing exposure to sunshine (37). Air pollution and high levels of air particles in big cities in Iran also diminish vitamin D synthesis because UV irradiation is absorbed by these particles and cannot reach the skin (38). However, in the current study we did not find any significant difference in vitamin D levels between rural and urban populations, which might be because of skin coverage in rural areas, or higher availability of vitamin D supplementation in cities.

The current study also reported a high prevalence of vitamin D deficiency independent of age. However, it is assumed that older individuals are more likely to be vitamin D deficient, not only because they may mostly stay indoors, but also because production of vitamin D in skin decreases with age (39). Nevertheless, there are other studies which reported that vitamin D deficiency was highly prevalent in adolescents (up to 70\%) (40) and even more in younger individuals (20). Female gender is another causal factor for vitamin D deficiency due to likelihood of pregnancy and lactation. Furthermore, greater body fat composition, which can lead to more vitamin D storage and consequently lower serum vitamin D, can increase vitamin D deficiency in women $(33,41)$. The present study did not find any significant difference between males and females. Considering the higher levels of concern held by women regarding their diet and health (42), higher consumption of vitamin D supplementation in women is conceivable. This could also explain a significantly reduced risk of vitamin $\mathrm{D}$ in females but not in males over the $12-\mathrm{y}$ period of 
our study. Another possible reason for not finding any significant difference in the prevalence of vitamin D deficiency between sub-groups of the study population might be due to the limited number of individuals in each, which limits the power to detect significant differences; however, the sample size was calculated to be large enough to determine if there were any significant changes in the prevalence of vitamin D deficiency from 2001 to 2013.

Vitamin D deficiency may be worsened as a result of secondary hyperparathyroidism caused by calcium inadequacy (43). Moreover, it has been shown that lower serum vitamin D levels were associated with specific genotypes (28). A well designed genome-wide association study revealed that different genes related to cholesterol metabolism, hydroxylation, and vitamin D transport affect vitamin D status and play roles in the etiology of vitamin D deficiency and insufficiency (44). This could be another possible reason for a higher prevalence of vitamin D deficiency in some populations, such as Iranians.

This study has a number of strengths. The first was its longitudinal design. There is limited information about the trend of vitamin D deficiency in longitudinal studies and thus this study is among the first that could assess this trend. This information is indispensable for health policies to prevent nutritional deficiencies and related diseases. Another strength is controlling for the possible confounders related to the prevalence and trend of vitamin D deficiency, including age, gender, BMI, region, season of blood collection and dietary intake of vitamin D. However, the current study also has some limitations. First, some of the characteristics of the study subjects differed significantly from that of the subjects in the total cohort. Overall, the study subjects were relatively younger and were in better health than the total cohort and, therefore, the study subjects may not be representative of the wider population. Second, although all study subjects were free from MetS in 2001, some developed the condition over the $12-y$ period and this may have influenced the present findings. However, after adjusting for the effect of the presence of MetS in the multivariate model, the results remained unaltered. Third, we do not have any data on vitamin D supplementation, PTH or calcium levels, which may have some influence on vitamin D levels and, therefore, the findings of the present study should be interpreted with caution.

In conclusion, the present study revealed that there was significant improvement in vitamin D status among Iranian adults over the $12-y$ period. However, the current prevalence of this nutrient deficiency is still high enough to be considered a significant public health issue. Considering the possible health consequences of vitamin D deficiency, there is an urgent need to develop population-wide strategies, such as supplementation and fortification, to prevent or control vitamin D deficiency. Further studies with representative samples are required to confirm the downward trends of vitamin D deficiency among Iranian adults, and to investigate the determinants of this deficiency in this population.

\section{Author's contribution}

H K-B contributed to the design of the study, analyzed the data and wrote the manuscript; MS, HR and AP collected the data. S-K N provided guidance in performing statistical analysis, and contributed to the interpretation of results and critical revision of the manuscript; NS contributed to the design of the study and critical revision of the manuscript. FA provided guidance on study design, and contributed to the interpretation of results and critical revision of the manuscript. All authors have read and approved the final manuscript.

\section{REFERENCES:}

1) Shaw NJ. 2004. Vitamin D deficiency rickets. In: Vitamin D and Rickets, Vol 6, p 93-104. Karger Publishers, London.

2) Vieth R, Kimball S, Hu A, Walfish PG. 2004. Randomized comparison of the effects of the vitamin D3 adequate intake versus $100 \mathrm{mcg}$ (4000 IU) per day on biochemical responses and the wellbeing of patients. Nutr J 3: 8 .

3) Nadir MA, Szwejkowski BR, Witham MD. 2010. Vitamin D and cardiovascular prevention. Cardiovasc Ther 28: e5-e12.

4) Holick MF. 2002. Vitamin D: the underappreciated D-lightful hormone that is important for skeletal and cellular health. Curr Opin Endocrinol Diabetes Obes 9: 87-98.

5) Holick MF. 2010. Vitamin D: Physiology, Molecular Biology, and Clinical Applications. Springer, New York.

6) Zittermann A, Iodice S, Pilz S, Grant WB, Bagnardi V, Gandini S. 2012. Vitamin D deficiency and mortality risk in the general population: a meta-analysis of prospective cohort studies. Am J Clin Nutr 95: 91-100.

7) Melamed ML, Michos ED, Post W, Astor B. 2008. 25-Hydroxyvitamin D levels and the risk of mortality in the general population. Arch Intern Med 168: 1629-1637.

8) Dobnig H, Pilz S, Scharnagl H, Renner W, Seelhorst U, Wellnitz B, Kinkeldei J, Boehm BO, Weihrauch G, Maerz W. 2008. Independent association of low serum 25-hydroxyvitamin D and 1,25-dihydroxyvitamin D levels with all-cause and cardiovascular mortality. Arch Intern Med 168: 1340-1349.

9) Food and Nutrition Board, Institute of Medicine. 2011. Dietary Reference Intakes for Calcium and Vitamin D. National Academies Press, Washington DC.

10) Gallagher JC, Sai AJ. 2010. Vitamin D insufficiency, deficiency, and bone health. J Clin Endocrinol Metab 95: 2630-2633.

11) Mithal A, Wahl D, Bonjour J-P, Burckhardt P, DawsonHughes B, Eisman J, Fuleihan GE-H, Josse R, Lips P, Morales-Torres J. 2009. Global vitamin D status and determinants of hypovitaminosis D. Osteoporos Int 20: 1807-1820.

12) Holick MF. 2007. Vitamin D deficiency. N Engl J Med 357: 266-281.

13) Ramphal L, Zhang J, Suzuki S. 2014. Ethnic disparities in the prevalence of the metabolic syndrome in American adults: data from the Examination of National Health and Nutrition Examination Survey 1999-2010. Proc (Bayl Uni Med Cent) 27: 92-95.

14) Quaggiotto P, Tran H, Bhanugopan M. 2014. Vitamin D deficiency remains prevalent despite increased labora- 
tory testing in New South Wales, Australia. Singapore Med J 55: 271.

15) Lips P. 2007. Vitamin D status and nutrition in Europe and Asia. J Steroid Biochem Mol Biol 103: 620-625.

16) Gaafar M, Badr S. 2013. An alarming high prevalence of vitamin D deficiency among healthy adults. Life Sci J 10: 3292-3298.

17) Ardawi M-S, Sibiany A, Bakhsh T, Qari M, Maimani A. 2012. High prevalence of vitamin D deficiency among healthy Saudi Arabian men: relationship to bone mineral density, parathyroid hormone, bone turnover markers, and lifestyle factors. Osteoporos Int 23: 675-686.

18) Heshmat R, Mohammad K, Majdzadeh S, Forouzanfar M, Bahrami A, Omrani GR, Nabipour I, Rajabian R, Hossein-Nezhad A, Hemami MR. 2008. Vitamin D deficiency in Iran: A multi-center study among different urban areas. Iran J Public Health 37(sup): 72-78.

19) Hossein-Nezhad A, Khoshniat N, Maghbooli M, Karimi Z, Mirzaei F, Hosseini A, Larijani B. 2009. Relationship between serum vitamin D concentration and metabolic syndrome among Iranian adults population. Daru 17: $1-5$.

20) Hovsepian S, Amini M, Aminorroaya A, Amini P, Iraj B. 2011. Prevalence of vitamin D deficiency among adult population of Isfahan City, Iran. J Health Popul Nutr 29: 149.

21) Sarrafzadegan N, Talaei M, Sadeghi M, Kelishadi R, Oveisgharan S, Mohammadifard N, Sajjadieh AR, Kabiri P, Marshall T, Thomas GN. 2011. The Isfahan cohort study: Rationale, methods and main findings. J Hum Hypertens 25: 545-553.

22) Sarrafzadegan N, Sadeghi M, Oveisgharan S, Iranipour R. 2013. Incidence of cardiovascular diseases in an Iranian population: the Isfahan Cohort Study. Arch Iran Med 16: 138.

23) Wang TJ, Pencina MJ, Booth SL, Jacques PF, Ingelsson E, Lanier K, Benjamin EJ, D’Agostino RB, Wolf M, Vasan RS. 2008. Vitamin D deficiency and risk of cardiovascular disease. Circulation 117: 503-511.

24) Hollis BW. 2008. Measuring 25-hydroxyvitamin D in a clinical environment: challenges and needs. Am J Clin Nutr 88: 507S-510S.

25) Sarraf-Zadegan N, Sadri G, Malek Afzali H, Baghaei M, Mohammadi Fard N, Shahrokhi S, Tolooie H, Pourmoghaddas M, Sadeghi M, Tavassoli A. 2003. Isfahan Healthy Heart Program: A comprehensive integrated community-based program for cardiovascular disease prevention and control. Acta Cardiol 58: 309-320.

26) Khosravi-Boroujeni H, Sarrafzadegan N, Mohammadifard N, Sajjadi F, Maghroun M, Asgari S, Rafieian-Kopaei M, Azadbakht L. 2013. White rice consumption and CVD risk factors among Iranian population. J Health Popul Nutr 31: 252-261.

27) Hanley JA, Negassa A, Forrester JE. 2003. Statistical analysis of correlated data using generalized estimating equations: an orientation. Am J Epidemiol 157: 364-375.

28) Baroncelli GI, Bereket A, El Kholy M, Audi L, Cesur Y, Ozkan B, Rashad M, Fernández-Cancio M, Weisman Y, Saggese G. 2008. Rickets in the Middle East: role of environment and genetic predisposition. J Clin Endocrinol Metab 93: 1743-1750.

29) Shahla A, Charehsaz S, Talebi R, Omrani M. 2005. Vitamin D deficiency in young females with musculoskel- etal complaints in Urmia, northwest of Iran. IJMS 30: 88-90.

30) Rahnavard Z, Eybpoosh S, Homami MR, Meybodi HA, Azemati B, Heshmat R, Larijani B. 2010. Vitamin D deficiency in healthy male population: Results of the Iranian multi-center osteoporosis study. Iran J Public Health 39: 45 .

31) Meerza D, Naseem I, Ahmed J. 2010. Can Vitamin D be a potential treatment for Type 2 diabetes mellitus. Diabetes Metab Syndr 4: 245-248.

32) Holick MF. 2004. Vitamin D: importance in the prevention of cancers, type 1 diabetes, heart disease, and osteoporosis. Am J Clin Nutr 79: 362-371.

33) Hagenau T, Vest R, Gissel T, Poulsen C, Erlandsen M, Mosekilde L, Vestergaard P. 2009. Global vitamin D levels in relation to age, gender, skin pigmentation and latitude: an ecologic meta-regression analysis. Osteoporos Int 20: 133-140.

34) Faurschou A, Beyer D, Schmedes A, Bogh M, Philipsen P, Wulf H. 2012. The relation between sunscreen layer thickness and vitamin D production after ultraviolet B exposure: a randomized clinical trial. Br J Dermatol 167 : 391-395.

35) Hiom S. 2006. Public awareness regarding UV risks and vitamin D-the challenges for UK skin cancer prevention campaigns. Prog Biophys Mol Biol 92: 161-166.

36) Aguado P, Del Campo M, Garces M, Gonzalez-Casaus M, Bernad M, Gijon-Banos J, Mola EM, Torrijos A, Martinez M. 2000. Low vitamin D levels in outpatient postmenopausal women from a rheumatology clinic in Madrid, Spain: their relationship with bone mineral density. Osteoporos Int 11: 739-744.

37) Puri S, Marwaha RK, Agarwal N, Tandon N, Agarwal R, Grewal K, Reddy D, Singh S. 2008. Vitamin D status of apparently healthy schoolgirls from two different socioeconomic strata in Delhi: relation to nutrition and lifestyle. Br J Nutr 99: 876-882.

38) Hosseinpanah F, Sima H, Heibatollahi M, Moghbel N, Asefzade S, Azizi F. 2010. The effects of air pollution on vitamin D status in healthy women: a cross sectional study. BMC Public Health 10: 519.

39) Holick M, Matsuoka L, Wortsman J. 1989. Age, vitamin D, and solar ultraviolet. Lancet 334: 1104-1105.

40) Moussavi M, Heidarpour R, Aminorroaya A, Pournaghshband Z, Amini M. 2005. Prevalence of vitamin D deficiency in Isfahani high school students in 2004. Horm Res 64: 144-148.

41) Dijkstra S, Van Beek A, Janssen J, De Vleeschouwer L, Huysman W, Van den Akker E. 2007. High prevalence of vitamin D deficiency in newborn infants of high-risk mothers. Arch Dis Child 92: 750-753.

42) Kiefer I, Rathmanner T, Kunze M. 2005. Eating and dieting differences in men and women. J Mens Health Gend 2: 194-201.

43) DeLucia MC, Mitnick ME, Carpenter TO. 2003. Nutritional rickets with normal circulating 25-hydroxyvitamin D: a call for reexamining the role of dietary calcium intake in North American infants. J Clin Endocrinol Metab 88: 3539-3545.

44) Wang TJ, Zhang F, Richards JB, Kestenbaum B, Van Meurs JB, Berry D, Kiel DP, Streeten EA, Ohlsson C, Koller DL. 2010. Common genetic determinants of vitamin D insufficiency: a genome-wide association study. Lancet 376: 180-188. 